\title{
Investigating Adherence to Medication in Patients Diagnosed with Systemic Lupus Erythematosus: A Cross-Sectional Study in Iran
}

\author{
Omid Eslami ${ }^{1}$, Amir Hossein Norooznezhad ${ }^{2}$, Shafieh Movassaghi ${ }^{3}$, Neda Naderi ${ }^{4}$, Abdolrahman Rostamian ${ }^{5}$, Taraneh \\ Dormohammadi Toosi ${ }^{3,6^{*}}$ \\ ${ }^{I}$ Gastroenterology and Hepatology Research Center, Institute of Basic and Clinical Physiology Sciences, Kerman \\ University of Medical Sciences, Kerman, Iran. Inflammation Research Center, Tehran University of Medical Sciences, \\ Tehran, Iran. ${ }^{3}$ Rheumatology Research center, Vali-Asr Hospital, Imam Khomeini Hospital Complex, Tehran University \\ of Medical Sciences, Tehran, Iran. ${ }^{4}$ Nephrology Research Center, Center of Excellence in Nephrology, Tehran University \\ of Medical Sciences, Tehran, Iran. ${ }^{5}$ Rheumatology Research center, Imam Khomeini Hospital, Tehran University of \\ Medical Sciences, Tehran, Iran. ${ }^{6}$ Rheumatology Division, University of San Diego, CA, USA.
}

\begin{abstract}
Systemic lupus erythematosus (SLE) is a systemic autoimmune disease with an unknown etiology. Although there is no definite treatment for this disease but using proper prescribed medications, mortality and morbidity ratios could be decreased.

The present study attempted to investigate adherence to medical treatment in patients suffering from SLE and to evaluate the related factors which influence adherence in order to improve patients' prognoses. In this cross-sectional study, 132 patients with SLE who referred to the rheumatology clinic of Imam Khomeini Hospital (Tehran, Iran) over the period of 2012-2013 were examined using a questionnaire. The applied questionnaire included two parts; the first part pertained to adherence to medication treatments (CQR: compliance questionnaire rheumatology), and the second part was related to the factors involved in adherence to medication treatments. The obtained data was analyzed using the Chi-Square test and processed by SPSS16.0 software. In this research, the mean adherence to drug treatment was calculated to be $72.48 \%$. Among the variables of age, marital status, salary, education, distance from the clinic (accessibility), disease duration, level of patient activity, main medications consumed, and side effects, the only significant association was found between adherence level and lack of side effects due to SLE ( $p<0.05)$. One hypothesis regarding cause of appropriate adherence to medical treatment in the studied group could be the hope of never experiencing side effects; however, more investigation is needed to prove this theory.
\end{abstract}

Keywords: Systemic lupus erythematosus, Treatment Adherence, Drug side effect

\section{Introduction}

Systemic Lupus Erythematosus (SLE) is an autoimmune disorder with an unknown etiology observed most frequently in women; however, young men and older people of both genders can also be affected [1]. Clinical symptoms include arthralgia and joint inflammation, skin rashes, photosensitivity, pleuritic or periodical chest pains, Raynaud's phenomenon, continuous fatigue, fever, and weight loss. According to the data, more than $60 \%$ of SLE patients present with symptoms in joints, skin [2,3], and kidney which is the msot commonly affected vital organ. A low number of patients show kidney involvement at the onset of SLE, but most patients manifest symptoms of kidney problems during the disease [4]. SLE's clinical manifestations are variable; thus, an exact diagnosis requires good clinical judgment, appropriate accuracy, and safe and acceptable laboratory tests. Although there is still no definite treatment for SLE, some methods, including training for patients, immunosuppressive drugs, and an exact follow-up program (for quick diagnosis of new clinical symptoms), are helpful. Moreover, treatment with glucocorticoids and immunosuppressive medications could decrease mortality and morbidity ratios among SLE patients [5].

Effective treatment for SLE requires supportive and medical interactions between patient and physician, but denying the disease or treatment and non-adherence to

Personal non-commercial use only. Rheumatology Research Journal. Copyright $($ C) 2020. All rights reserved

*Corresponding Author: Taraneh Dormohammadi Toosi, M.D. Postal address: Rheumatology Research center, Imam Khomeini Hospital Complex, Keshavarz Blvd, Tehran, Iran. E-mail: taraneh_dormohammadi@yahoo.com_P. O. Box: 1419733141, Tell: +98 2161190

Received: 02 November 2019; Accepted: 01 December 2019 
medications could occur because of occupational or life problems [6, 7]. Because SLE has relapse episodes, appropriate follow up by the patient could be effective in decreasing the attacks or even quickening the response to treatment [8]. Adherence to medication refers to "an individual's behavior in consuming medications and following suggested changes in a diet or even in lifestyle pattern". Developing this term could reflect changes in views, indicating that the patient needs to be more involved in treatment decision-making [9]. In a study performed in the United States on the factors determining adherence to medical treatments, it was shown that financial problems, fear of side effects, problems in accessing public health systems, and lack of adequacy are of the most important barriers to treatment adherence [10]. Furthermore, patients are concerned about the safety of their drugs in the long term, which may influence treatment adherence. Other potent barriers to adherence to medication are noted to be depression, short-term memory disorder, disease symptoms, drug side effects, and lack of trust in one's physician [11]. Adherence to medication therapy could be evaluated by direct or indirect methods. In direct methods, adherence to medical treatment is explored through direct observation and laboratory evaluation of serum levels of the SLE drugs, but it cannot be used in many cases due to limitations. Indirect methods are introduced as tablet counting, electronic recording (by specific boxes recording the number of opening), and self-expression techniques (e.g., CQR) [12].

The present study has attempted to investigate adherence to medication in patients diagnosed with SLE. Detecting any possible effective factor in patient adherence was also considered as an important goal of the present study in order to determine its worthiness and ability to improve patient's prognosis by modifying any identified factor.

\section{Materials and Methods}

\section{Study design}

The present cross-sectional study was conducted on patients diagnosed with SLE based on American College of Rheumatology (ACR) criteria [13] in the Rheumatology Clinic of Imam Khomeini Hospital Complex (Tehran, Iran) over the period of June 2012-May 2013. This study was approved by the Medical Ethics Committee of Tehran University of Medical Sciences.

\section{Measurements}

Patients' demographic information, such as age, gender, average salary, marital status, age, distance from the clinic (accessibility), education, disease duration, and duration of medication administration, were recorded. Then adherence to medication treatment was evaluated by completing a questionnaire based on the validated $\mathrm{CQR}$ questionnaire
[14]. The applied questionnaire entailed 19 items with a maximum score of 100 . The results of the questionnaire were classified into good following (76-100), average following (51-75), weak following (26-50), and very weak following (0-25). The reliability of the questionnaire was evaluated using alpha Cronbach (on 10 patients with SLE). Other questions were designed based on secondary research objectives. The systematic lupus erythematosus disease activity index (SLEDAI) was used to investigate the amount of disease activity [15]. Based on the SLEDAI system, the patients were divided into 5 groups: without disease activity $($ SLEDAI $=0)$, mild disease activity $($ SLEDAI $=1-5)$, average disease activity $($ SLEDAI = 6-10), severe disease activity (SLEDAI $=11-19$ ), and very severe disease activity $($ SLEDAI $=20)$ [16]. The collected information was analyzed using SPSS 16.0 software (SPSS Inc., Chicago, IL, USA). The response rate of the participants was $100 \%$.

\section{Statistical Analysis}

All categorical variables were presented as N (\%). Twosided Chi-square/Fisher's exact tests were used to assess the associations between two categorical variables. Significance level was considered to be $p$ value $<0.05$.

\section{Results}

The present study investigated 132 patients $(100 \%$ response rate) including 122 females (92.4\%) and 10 males (7.6\%) suffering from SLE; 95 patients (72\%) were married and the other $37(28 \%)$ were single (details in Table 1). The mean of adherence to drug treatment was calculated to be $72.48 \%$.

The details of biodemographic, social, and economical status of the patients according to their level of adherence is provided in Table 1. No significant association was found between level of adherence and variables such as gender, marital status, distance from the clinic (accessibility), education, disease duration, or duration of drug taking.

As shown in Table 2, no significant association was found between the level of disease activity (based on SLEDAI criteria) and adherence to medication treatment. Thus, it was shown that being at different levels of severity of SLE does not affect medication adherence ( $p$ value $=$ $0.640)$, but overall, most of the patients showed a moderate level of compliance $(51-75 \%)$, especially patients in the severe group. Moreover, no significant association was found between adherence to drug treatment and various drugs consumed ( $p$ value $=0.767)$ and duration of usage ( $p$ value $=0.627)$. 
Table 1. Details of the biodemographic and socioeconomical status of the patients according to their levels of adherence to their treatments.

\begin{tabular}{|c|c|c|c|c|c|}
\hline \multirow{2}{*}{ Variable } & & \multicolumn{2}{|c|}{ Compliance (\%) } & \multirow{2}{*}{ Total } & \multirow{2}{*}{ Pvalue } \\
\hline & & {$[50-75)$} & $(75-100]$ & & \\
\hline \multirow{2}{*}{ Sex } & Female & $66(58.4 \%)$ & $47(41.6 \%)$ & $113(100.0 \%)$ & \multirow{2}{*}{0.47} \\
\hline & Male & $7(70.0 \%)$ & $3(30.0 \%)$ & $10(100.0 \%)$ & \\
\hline \multirow{3}{*}{ Marital status } & Single & $51(56.7 \%)$ & $39(43.3 \%)$ & $90(100.0 \%)$ & \multirow{2}{*}{0.31} \\
\hline & Married & $22(66.7 \%)$ & $11(33.3 \%)$ & $33(100.0 \%)$ & \\
\hline & {$[15-25)$} & $19(63.3 \%)$ & $11(36.7 \%)$ & $30(100.0 \%)$ & \multirow{4}{*}{0.06} \\
\hline \multirow{3}{*}{ Age (years) } & {$[25-35)$} & $33(66.0 \%)$ & $17(34.0 \%)$ & $50(100.0 \%)$ & \\
\hline & {$[35-45)$} & $8(34.8 \%)$ & $15(65.2 \%)$ & $23(100.0 \%)$ & \\
\hline & $45 \geq$ & $13(65.0 \%)$ & $7(35.0 \%)$ & $20(100.0 \%)$ & \\
\hline \multirow{5}{*}{ Salary } & Quarter 1 & $21(61.8 \%)$ & $13(38.2 \%)$ & $34(100.0 \%)$ & \multirow{4}{*}{0.87} \\
\hline & Quarter 2 & $30(58.8 \%)$ & $21(41.2 \%)$ & $51(100.0 \%)$ & \\
\hline & Quarter 3 & $14(53.8 \%)$ & $12(46.2 \%)$ & $26(100.0 \%)$ & \\
\hline & Quarter 4 & $8(66.7 \%)$ & $4(33.3 \%)$ & $12(100.0 \%)$ & \\
\hline & None & $7(43.8 \%)$ & $9(56.3 \%)$ & $16(100.0 \%)$ & \multirow{5}{*}{0.15} \\
\hline \multirow{3}{*}{ Education (years) } & {$[1-6)$} & $8(47.1 \%)$ & $9(52.9 \%)$ & $17(100.0 \%)$ & \\
\hline & {$[6-12)$} & $42(63.6 \%)$ & $24(36.4 \%)$ & $66(100.0 \%)$ & \\
\hline & {$[12-14)$} & $8(88.9 \%)$ & $1(11.1 \%)$ & $9(100.0 \%)$ & \\
\hline \multirow{6}{*}{$\begin{array}{l}\text { Distance to the clinic } \\
\qquad(\mathrm{Km})\end{array}$} & $14 \geq$ & $73(59.3 \%)$ & $50(40.7 \%)$ & $123(100.0 \%)$ & \\
\hline & $<5$ & $3(100.0 \%)$ & $0(.0 \%)$ & $3(100.0 \%)$ & \multirow{5}{*}{0.15} \\
\hline & {$[5-10)$} & $9(60.0 \%)$ & $6(40.0 \%)$ & $15(100.0 \%)$ & \\
\hline & {$[10-50)$} & $7(36.8 \%)$ & $12(63.2 \%)$ & $19(100.0 \%)$ & \\
\hline & {$[50-100)$} & $17(56.7 \%)$ & $13(43.3 \%)$ & $30(100.0 \%)$ & \\
\hline & $100 \geq$ & $37(66.1 \%)$ & $19(33.9 \%)$ & $56(100.0 \%)$ & \\
\hline \multirow{4}{*}{$\begin{array}{c}\text { Disease duration } \\
\text { (years) }\end{array}$} & $1<$ & $14(77.8 \%)$ & $4(22.2 \%)$ & $18(100.0 \%)$ & \multirow{4}{*}{0.10} \\
\hline & {$[1-5)$} & $26(48.1 \%)$ & $28(51.9 \%)$ & $54(100.0 \%)$ & \\
\hline & {$[5-10)$} & $15(68.2 \%)$ & $7(31.8 \%)$ & $22(100.0 \%)$ & \\
\hline & $10 \geq$ & $18(62.1 \%)$ & $11(37.9 \%)$ & $29(100.0 \%)$ & \\
\hline \multirow{4}{*}{$\begin{array}{l}\text { Duration of drug } \\
\text { consumption } \\
\text { (months) }\end{array}$} & $6<$ & $8(57.1 \%)$ & $6(42.9 \%)$ & $14(100.0 \%)$ & \multirow{4}{*}{0.62} \\
\hline & {$[6-12)$} & $7(77.8 \%)$ & $2(22.2 \%)$ & $9(100.0 \%)$ & \\
\hline & {$[12-24)$} & $8(66.7 \%)$ & $4(33.3 \%)$ & $12(100.0 \%)$ & \\
\hline & $24 \geq$ & $50(56.8 \%)$ & $38(43.2 \%)$ & $88(100.0 \%)$ & \\
\hline
\end{tabular}


Table 2. The prevalence of adherence to medication treatment and the level of disease activity in the patients diagnosed.

\begin{tabular}{|c|c|c|c|c|}
\hline \multirow[b]{2}{*}{ Disease activity } & \multicolumn{2}{|c|}{ Compliance score } & \multirow{2}{*}{ Total compliance } & \multirow[b]{2}{*}{$P$ value } \\
\hline & {$[50-75)$} & [75-100] & & \\
\hline Not activity & $43(57.3 \%)$ & $32(42.7 \%)$ & $75(100.0 \%)$ & \\
\hline Mild & $43(57.3 \%)$ & $32(42.7 \%)$ & $75(100.0 \%)$ & \\
\hline Moderate & $7(53.8 \%)$ & $6(46.2 \%)$ & $13(100.0 \%)$ & 0.64 \\
\hline Severe & $5(83.3 \%)$ & $1(16.7 \%)$ & $6(100.0 \%)$ & \\
\hline Total & $73(59.3 \%)$ & $50(40.7 \%)$ & $123(100.0 \%)$ & \\
\hline
\end{tabular}

As can be seen in Table 3, the association between adherence to drug treatment and the side effects of Lupus disease was investigated, it was revealed that there is no significant association between the two variables. The association between adherence to drug treatment and lack of side effects of Lupus disease was significant $(p$ value $=0.029$ ).

Table 3. The prevalence of adherence to drug treatment and presented side effect(s) in the patients.

\begin{tabular}{|c|c|c|c|c|c|}
\hline \multicolumn{6}{|c|}{ Compliance } \\
\hline \multicolumn{2}{|c|}{ Presented side effect(s) } & \multicolumn{2}{|c|}{ Compliance } & Total & P value \\
\hline \multirow{3}{*}{ CNS } & Presented & $8(66.7 \%)$ & $4(33.3 \%)$ & $12(100.0 \%)$ & \multirow{3}{*}{0.58} \\
\hline & & & & & \\
\hline & Not presented & $65(58.6 \%)$ & $46(41.4 \%)$ & $111(100.0 \%)$ & \\
\hline \multirow{3}{*}{ Renal } & Presented & $31(59.6 \%)$ & $21(40.4 \%)$ & 52 (100.0\%) & \multirow{3}{*}{0.90} \\
\hline & & & & & \\
\hline & Not presented & $42(59.2 \%)$ & $29(40.8 \%)$ & $71(100.0 \%)$ & \\
\hline \multirow{3}{*}{ Cardiac } & Presented & $5(100.0 \%)$ & $0(0.0 \%)$ & $5(100.0 \%)$ & \multirow{3}{*}{0.07} \\
\hline & & & & & \\
\hline & Not presented & $68(57.6 \%)$ & $50(42.4 \%)$ & $118(100.0 \%)$ & \\
\hline \multirow{3}{*}{ Hematologic } & Presented & $15(57.7 \%)$ & $11(42.3 \%)$ & $26(100.0 \%)$ & \multirow{3}{*}{0.80} \\
\hline & & & & & \\
\hline & Not presented & $58(59.8 \%)$ & $39(40.2 \%)$ & $97(100.0 \%)$ & \\
\hline \multirow{3}{*}{ Arthritis } & Presented & $33(66.0 \%)$ & $17(34.0 \%)$ & $50(100.0 \%)$ & \multirow{3}{*}{0.26} \\
\hline & & & & & \\
\hline & Not presented & $40(54.8 \%)$ & $33(45.2 \%)$ & $73(100.0 \%)$ & \\
\hline \multirow{3}{*}{ Pulmonary } & Presented & $6(66.7 \%)$ & $3(33.3 \%)$ & $9(100.0 \%)$ & \multirow{3}{*}{0.64} \\
\hline & & & & & \\
\hline & Not presented & $67(58.8 \%)$ & $47(41.2 \%)$ & $114(100.0 \%)$ & \\
\hline \multirow{3}{*}{ Gastrointestinal } & Presented & $4(100.0 \%)$ & $0(0.0 \%)$ & $4(100.0 \%)$ & \multirow{3}{*}{0.14} \\
\hline & & & & & \\
\hline & Not presented & $69(58.0 \%)$ & $50(42.0 \%)$ & $119(100.0 \%)$ & \\
\hline \multirow{2}{*}{$\begin{array}{l}\text { Other side } \\
\text { effect(s) }\end{array}$} & Presented & $8(38.1 \%)$ & $13(61.9 \%)$ & $21(100.0 \%)$ & \multirow{2}{*}{0.02} \\
\hline & Not presented & $65(63.7 \%)$ & $37(36.3 \%)$ & $102(100.0 \%)$ & \\
\hline
\end{tabular}




\section{Discussion}

The current study enrolled 132 patients diagnosed with SLE (92.4\% females). The number of females dominated over that of males, aligning with other studies [17]. The calculated mean adherence to medication was $72.48 \%$, which is similar to the findings obtained by GarciaGonzalez et al. [18]. The current study found no significant association between adherence to treatment and marital status, although Koneru et al. [19] reported that being single was an effective factor in decreasing adherence to treatment. Oliveira et al. [20] stated that family support was a positive factor in increasing adherence to medication therapy. The most significant difference between the present study and other studies may be due to cultural differences between the studied countries. In Iran, single individuals are sufficiently supported by their families both emotionally and even financially throughout their lives. As mentioned, no significant association was found between adherence to drug treatment and major organ damage. On the other hand, there was a significant association between adherence to drug treatment and lack of SLE side effects. In fact, patients who did not experience Lupus side effects had greater adherence. It could be hypothesized that end organ damage could decrease patients' morale in continuing the proper treatment process and also decrease one's assurance in prescribed medications. As the data showed, the most frequent side effect of SLE was kidney involvement, which is consistent with the findings reported by Ortega et al. [4]. As Oliveira et al. [20], GarciaGonzalez et al. [18], and Mosley-Williams et al. [11] reported, patients with joint involvement or musculocutaneous manifestations showed lower adherence to medication protocols, which differed from the current results. the present study found no significant association between patient's financial status and adherence to treatment, which is consistent with the conclusion drawn by Chambers et al. [21], while studies done by GarciaGonzalez et al. [18] and Oliveira et al. [20] revealed that financial problems comprised one of the barriers to adherence to treatment. Such differing results may be due to dissimilar treatment costs in various countries and varying government and insurance company support. Although the present study found no significant association between distance from the clinic (accessibility) and treatment adherence, patients who live $5 \mathrm{~km}$ from the clinic showed an average adherence to treatment, which is somewhat similar to the findings of Uribe AG et al. [7]. In their study, long duration of disease, distance from the clinic (accessibility), and more severe disease activity were the factors which predicted lack of adherence to treatment.

The current study found no significant association between duration of medication administration and adherence. It seems that no study has observed a significant association between the effect of duration of drug administration and adherence to drug treatment, which could perhaps be explained by the small sample sizes and average adherence to treatment. The findings of this study also indicated that there was no significant association between adherence to treatment and various drugs. Although there was no significant association between adherence to treatment and disease duration in this study, patients suffering with SLE for less than one year showed less adherence compared to others. This is inconsistent with the findings of the research carried out by Urib AG et al. [7], possibly because of the different methods of investigating adherence to treatment. One of the reasons for lower adherence to drug treatment among these patients may be denial of their illness and the usefulness of drugs in controlling their symptoms and disease.

The current study experienced some limitations, such as the method of evaluating adherence to medication, though attempts were made to choose the best, validated questionnaire, and the lack of low-compliance patients who did not follow their visit program. The low number of involved patients and being a multi-center study could be considered as other limitations.

\section{Conclusion}

The present research showed that more than half of the patients suffering from SLE had average or weak adherence to treatments, which could be resulted from the development of side effects of the disease over the long term or the imposition of financial and mental costs on society. Proper patient education and the attraction of familial support can result in an increased adherence to treatment. One of the most important underlying causes of weak or even average adherence to treatment for SLE or any other disease is a lack of appropriate education about the patients' illnesses. As a result, the authors of this study recommend that a well-designed training program be established for SLE patients to improve their compliance with treatment protocols.

\section{Conflict of interest}

The authors report that they have no conflicts of interest to declare.

\section{References}

1. Nusbaum JS, Mirza I, Shum J, Freilich RW, Cohen RE, Pillinger MH. et al. Sex Differences in Systemic Lupus Erythematosus: Epidemiology, Clinical Considerations, and Disease Pathogenesis. Mayo Clin Proc 2020; 95(2):384-94. doi: 10.1016/j.mayocp.2019.09.012.
2. Heinlen LD, McClain MT, Merrill J, Akbarali YW, Edgerton CC, Harley JB. et al. Clinical criteria for systemic lupus erythematosus precede diagnosis, and associated autoantibodies are present before clinical symptoms. Arthritis Rheum 2007; 56(7):2344-51. doi: 10.1002/art.22665. 
3. Nossent J, Kiss E, Rozman B, Pokorny G, Vlachoyiannopoulos $\mathrm{P}$, Olesinska $\mathrm{M}$. et al. Disease activity and damage accrual during the early disease course in a multinational inception cohort of patients with systemic lupus erythematosus. Lupus 2010; 19(8):949-56. doi: 10.1177/0961203310366572.

4. Ortega LM, Schultz DR, Lenz O, Pardo V, Contreras GN. Review: Lupus nephritis: pathologic features, epidemiology and a guide to therapeutic decisions. Lupus 2010; 19(5):557-74. doi: 10.1177/0961203309358187.

5. Lam NC, Ghetu MV, Bieniek ML. Systemic Lupus Erythematosus: Primary Care Approach to Diagnosis and Management. Am Fam Physician 2016; 94(4):284-94.

6. Kumar K, Chambers S, Gordon C. Challenges of ethnicity in SLE. Best Pract Res Clin Rheumatol 2009; 23(4):54961. doi: 10.1016/j.berh.2009.04.005.

7. Uribe AG, Alarcón GS, Sanchez ML, McGwin G Jr., Sandoval R, Fessler BJ. et al. Systemic lupus erythematosus in three ethnic groups. XVIII. Factors predictive of poor compliance with study visits. Arthritis Rheum 2004; 51(2):258-63. doi: 10.1002/art.20226.

8. Danowski A, Magder L, Petri M. Flares in lupus: Outcome Assessment Trial (FLOAT), a comparison between oral methylprednisolone and intramuscular triamcinolone. $\boldsymbol{J}$ Rheumatol 2006; 33(1):57-60.

9. Treharne GJ, Lyons AC, Hale ED, Douglas KM, Kitas GD. 'Compliance' is futile but is 'concordance' between rheumatology patients and health professionals attainable? Rheumatology (Oxford) 2006; 45(1):1-5. doi: 10.1093/rheumatology/kei223.

10. Garcia Popa-Lisseanu MG, Greisinger A, Richardson M, O'Malley KJ, Janssen NM, Marcus DM. et al. Determinants of treatment adherence in ethnically diverse, economically disadvantaged patients with rheumatic disease. J Rheumatol 2005; 32(5):913-19.

11. Mosley-Williams A, Lumley MA, Gillis M, Leisen J, Guice D. Barriers to treatment adherence among African American and white women with systemic lupus erythematosus. Arthritis Rheum 2002; 47(6):630-38. doi: 10.1002/art.10790.

12. de Achaval S, Suarez-Almazor ME. Treatment adherence to disease-modifying antirheumatic drugs in patients with rheumatoid arthritis and systemic lupus erythematosus. Int J Clin Rheumtol 2010; 5(3):313-26. doi: 10.2217/ijr.10.15.
13. Guidelines for referral and management of systemic lupus erythematosus in adults. American College of Rheumatology Ad Hoc Committee on Systemic Lupus Erythematosus Guidelines. Arthritis Rheum 1999; 42(9):1785-96. doi: 10.1002/15290131(199909)42:9<1785::aid-anr1>3.0.co;2-\#.

14. de Klerk E, van der Heijde D, Landewé R, van der Tempel H, van der Linden S. The compliancequestionnaire-rheumatology compared with electronic medication event monitoring: a validation study. $\boldsymbol{J}$ Rheumatol 2003; 30(11):2469-75.

15. Yee CS, Isenberg DA, Prabu A, Sokoll K, Teh LS, Rahman A. et al. BILAG-2004 index captures systemic lupus erythematosus disease activity better than SLEDAI2000. Ann Rheum Dis 2008; 67(6):873-76. doi: 10.1136/ard.2007.070847.

16. Mosca M, Bombardieri S. Assessing remission in systemic lupus erythematosus. Clin Expl Rheumatol 2006; 24(6 Suppl 43):S-99-104.

17. Tan TC, Fang H, Magder LS, Petri MA. Differences between male and female systemic lupus erythematosus in a multiethnic population. J Rheumatol 2012; 39(4):75969. doi: 10.3899/jrheum.111061.

18. Garcia-Gonzalez A, Richardson M, Garcia PopaLisseanu M, Cox V, Kallen MA, Janssen N. et al. Treatment adherence in patients with rheumatoid arthritis and systemic lupus erythematosus. Clin Rheumatol 2008; 27(7):883-89. doi: 10.1007/s10067-007-0816-6.

19. Koneru S, Kocharla L, Higgins GC, Ware A, Passo MH, Farhey YD. et al. Adherence to medications in systemic lupus erythematosus. J Clin Rheumatol 2008; 14(4):19501. doi: 10.1097/RHU.0b013e31817a242a.

20. Oliveira-Santos M, Verani JF, Klumb EM, Albuquerque EM. Evaluation of adherence to drug treatment in patients with systemic lupus erythematosus in Brazil. Lupus 2011; 20(3):320-29. doi: 10.1177/0961203310383736.

21. Chambers SA, Raine R, Rahman A, Isenberg D. Why do patients with systemic lupus erythematosus take or fail to take their prescribed medications? A qualitative study in a UK cohort. Rheumatology (Oxford) 2009; 48(3):26671. doi: $10.1093 /$ rheumatology/ken479. 